\title{
Potential of high-frequency ultrasonography in the management of atopic dermatitis
}

\author{
MARA SABĂU ${ }^{1 *}$, ANDREEA NICOLETA BOCA ${ }^{2 *}$, \\ ROXANA FLAVIA ILIES ${ }^{3 *}$ and ALEXANDRU TĂTARU ${ }^{1}$
}

\begin{abstract}
Departments of ${ }^{1}$ Dermatology, ${ }^{2}$ Pharmacology, Toxicology and Clinical Pharmacology, and ${ }^{3}$ Faculty of Medicine, 'Iuliu Hatieganu' University of Medicine and Pharmacy, 400423 Cluj-Napoca, Romania
\end{abstract}

Received July 6, 2018; Accepted August 24, 2018

DOI: $10.3892 / \mathrm{etm} .2018 .6984$

\begin{abstract}
Atopic dermatitis (AD) is a chronic, highly pruritic skin disorder, with a broad spectrum of clinical manifestations, characterized by abnormal skin barrier function. High-frequency ultrasonography (HF-USG) is an instrument with potential use in a variety of skin conditions. Previous studies on HF-USG in AD reported that apparently non-lesional skin also presents barrier defects. We aimed to assess lesional and non-lesional skin of AD patients with the use of HF-USG, focusing on skin barrier function and inflammation. We included a group of AD patients and a control group. On both we performed analysis with the use of HF-USG. The thickness of the subepidermal low-echogenic band (SLEB) and the skin echogenity were recorded for active lesions, as well as non-lesional skin in all subjects included. For AD patients the clinical severity was measured using SCORing Atopic Dermatitis and correlated with Dermatology Life Quality Index (DLQI). The mean thickness of the hypoechoic band was wider in the lesional skin of AD. In the non-lesional skin of AD patients SLEB was identified to appear, but it was thinner. The skin echogenity of the control group was higher than that of AD patients. Our results indicate that skin ultrasonography is able to assess specific modifications of the AD skin. A hypoechoic band in the non-lesional skin of some AD patients may indicate subclinical eczematous lesions.
\end{abstract}

Correspondence to: Dr Andreea Nicoleta Boca, Department of Pharmacology, Toxicology and Clinical Pharmacology, 33 Mihai Veliciu Street, 400423 Cluj-Napoca, Romania

E-mail: boca.andreea@umfcluj.ro

*Contributed equally

Abbreviations: AD, atopic dermatitis; HF-USG, high-frequency ultrasonography; SLEB, subepidermal low-echogenic band; DLQI, Dermatology Life Quality Index; QoL, Quality of Life; SCORAD, SCORing Atopic Dermatitis

Key words: atopic dermatitis, high-frequency ultrasonography, subepidermal low-echogenic band, quality of life, severity

\section{Introduction}

Atopic dermatitis (AD) is a chronic, pruritic skin condition that occurs most frequently in children, but can also affect adults. It is a complex, multifactorial, inflammatory disease; it affects an increasing number of patients and follows a relapsing course (1).

Patients with AD have a broad spectrum of clinical manifestations and a personal or family history of type I allergies (allergic rhinitis, asthma) associated with elevated serum immunoglobulin E (IgE) levels.

During the past 3 decades, the incidence of AD has clearly increased, especially in industrialized countries. Nowadays the prevalence is $15-30 \%$ in children and $2-10 \%$ in adults, and AD is becoming one of the most common chronic skin disorders in developed countries, with an important socio-economic impact (2).

This cutaneous disease has a major impact on patients' quality of life (QoL), due to the intense pruritus, sleep loss, dietary restrictions and the psychosocial boundaries it creates (3).

AD ethiopathogenesis and its causative factors remain a matter of debate, despite the numerous studies carried out. Epidermal barrier abnormalities and gene-environment interactions contribute to the clinical heterogenicity of AD, from dry skin to infection and severe erythroderma (4).

Recent studies reveal that clinically normal-appearing skin may present minimal inflammation with a sparse perivascular T-cell infiltrate. Langerhans cells with surface-bound IgE are likely to be present, even if to a lesser extent than within affected regions. Disturbed barrier function due to dehydration and reduced stratum corneum (SC) were detected in clinically healthy skin. These facts substantiate the need for dermatologists to consider the correct evaluation and therapeutic management of apparently healthy skin, along with lesional sites (5).

QoL assessment has become an important tool in evaluating the impact of chronic conditions such as eczema (6). It is currently considered an important outcome measure in clinical trials and practice, assessing the disease progression and the response to treatment. Patients' QoL can be influenced by many factors and is mainly related to the AD symptoms. The most common and life-impacting one is pruritus, a major complaint 
in most AD cases. This may impact the psychosomatic aspect of the condition and deserves special consideration during therapeutic management.

The Dermatology Life Quality Index (DLQI) is one of the most common instruments used in research and general practice for measuring QoL (7).

For clinical evaluation of the cutaneous lesions during all therapeutic phases it is mandatory to determine the severity of AD. The European Task Force on Atopic Dermatitis (ETFAD) has developed the SCORing AD (SCORAD). This established a consensus on assessment methods for $\mathrm{AD}$, making possible the comparison between study results (8). There are studies showing that AD impacts negatively on the QoL, proportional to the severity of the disease (9).

High-frequency ultrasonography (HF-USG) has been used in dermatology since the 1970s. It has been consistently improved and nowadays it offers the clinician the opportunity of real-time imaging, with the possibility of various measurements of morphological, physiological and pathological aspects. The images reflect the structure and tissue composition, influenced by the properties of the specific sound wave. Echogenicity of the dermis is one of the main relevant parameters, influenced by several factors. It is determined by orientation of collagen fibers, ground substance and water content (10-12).

An increase in echogenicity is caused by diseases with accumulation of fibers, besides increased number of inflammatory or neoplastic cells. Fiber damage will lower the echogenicity of the dermis. In inflammatory dermatoses, such as AD, it is easy to detect a subepidermal low-echogenic band (SLEB).

A main ultrasonographic characteristic of AD is the SLEB, accompanied by lower echogenicity of the other layers of the skin, compared to healthy skin. The SLEB is observed mainly due to edema and inflammatory cell infiltration has been reported in correlation with disease severity. It could be used as a major parameter for monitoring the treatment efficacy (13).

In light of these facts, the present study aims to assess lesional and non-lesional skin of AD patients with the use of HF-USG, focusing on skin barrier function and inflammation, quantified by the SLEB. In non-lesional skin, a hypoechoic band could indicate a subclinical eczematous reaction, anticipating the typical skin lesions.

\section{Patients and methods}

Participants. We included in our study a group of 10 patients, 8 women and 2 men. They joined the study on a volunteer basis. They were diagnosed with AD in accordance with the Hanifin and Rajka criteria (14), with a minimum of one active lesion at the time of the study. We excluded patients with uncertain diagnoses, as well as patients with overlapping conditions (for instance, an overlap of AD and systemic lupus erythematosus), which could impact overall skin health. Patients were of different ages, ranging from 15 to 74 years.

The patients were referred to the Department of Dermatology, part of Iuliu Hatieganu University of Medicine and Pharmacy, or to DEA Clinic, due to the exacerbation of skin lesions in the period from November 2017 to April 2018. We performed a full skin examination, searching for clinical signs of $\mathrm{AD}(15)$. We noted that patients presented marked xerosis and typical lesions of AD: facial pallor, keratosis pilaris, palmar hyperliniarity, periocular manifestations, lichenification, prurigo and pitiriazis alba.

The study was designed as a matched case-control study, where each patient is its own control, by choosing two homologous areas for HF-USG: one with an active dermatitis lesion and one deemed clinically healthy. The study was approved by the Ethics Committee of 'Iuliu Hatieganu' University of Medicine and Pharmacy (Cluj-Napoca, Romania). The patients were informed of their role in the study, the data that was collected during the study, and signed informed consents to participate in the study, and for the study results to be published.

Clinical evaluation. Each patient was evaluated clinically with regards to the severity, extent and psychological impact of the disease. We assessed the clinical severity of AD using SCORAD. We registered the patients, age, weight, height, and medical conditions (16)

Patients experience a variety of symptoms due to AD, these may affect their QoL. DLQI was used to quantify the impact of AD on the patients' QoL (17).

We performed HF-USG, focusing on the measurements on the thickness and intensity of the dermis, and the SLEB, both in apparently healthy skin, and in an active patch of AD. For this we used the DermaLab Combo ${ }^{\circledR}$ device (Cortex Technology, Hadsund, Denmark) (13).

Statistical analysis. The descriptive statistical analysis was performed in Microsoft Excel (Microsoft, Redmond, WA, USA), while the inferential statistical analysis was carried out using MedCalc Statistical Software version 18.2.1 (MedCalc Software bvba, Ostend, Belgium). To describe continuous variables, the median and 25 and 75 th percentiles were used. To determine the correlations between variables, Spearman's rho coefficient test was used.

\section{Results}

Study group features. Our study group of 10 subjects included 8 female patients and 2 male patients, aged between 15 and 74 years. The median age was 26 years, with the 25th percentile at 21.75 and the 75 th percentile at $32.5,20 \%$ of patients had a BMI over 30, while another $20 \%$ had a BMI under 18. The rest of the patients were of normal weight, with a BMI between 18 and 25. The majority of patients included in our study were young adults, with normal BMI.

Skin assessment. The Fitzpatrick phototype distribution was as follows: Patients (10\%) were phototype I, 60\% phototype II and $30 \%$ phototype III. According to our geographic area, most of the patients included, had the phototype specific for our region.

All the patients reported xerosis, with $80 \%$ reporting facial pallor, $50 \%$ keratosis pilaris, $50 \%$ palmar hyperliniarity and $50 \%$ periocular, perioral or periauricular manifestations, $30 \%$ reported prurigo, $20 \%$ manifested lichenification of the skin and $10 \%$ pitiriasis alba The reported clinical manifestations are depicted in Fig. 1. 
Table I. Correlation between DLQI, SCORAD and HF-USG parameters.

\begin{tabular}{lccccc}
\hline Tests & DLQI & SCORAD & Lesional LEB & Lesional thickness & Lesional intensity \\
\hline $\begin{array}{l}\text { DLQI } \\
\begin{array}{l}\text { Spearman's rho } \\
\text { correlation coefficient }\end{array}\end{array}$ & 1.000 & 0.474 & -0.349 & 0.109 & 0.024 \\
$\begin{array}{l}\text { P-value } \\
\text { SCORAD }\end{array}$ & $<0.001$ & 0.166 & 0.324 & 0.763 & 0.947 \\
$\begin{array}{l}\text { Spearman's rho } \\
\text { correlation coefficient }\end{array}$ & 0.474 & 1.000 & 0.177 & -0.309 & -0.515 \\
\begin{tabular}{l} 
P-value \\
\hline
\end{tabular} & 0.166 & $<0.001$ & 0.625 & 0.385 & 0.0128 \\
\hline
\end{tabular}

DLQI, Dermatology Life Quality Index; SCORAD, SCORing Atopic Dermatitis; HF-USG, high-frequency ultrasonography.

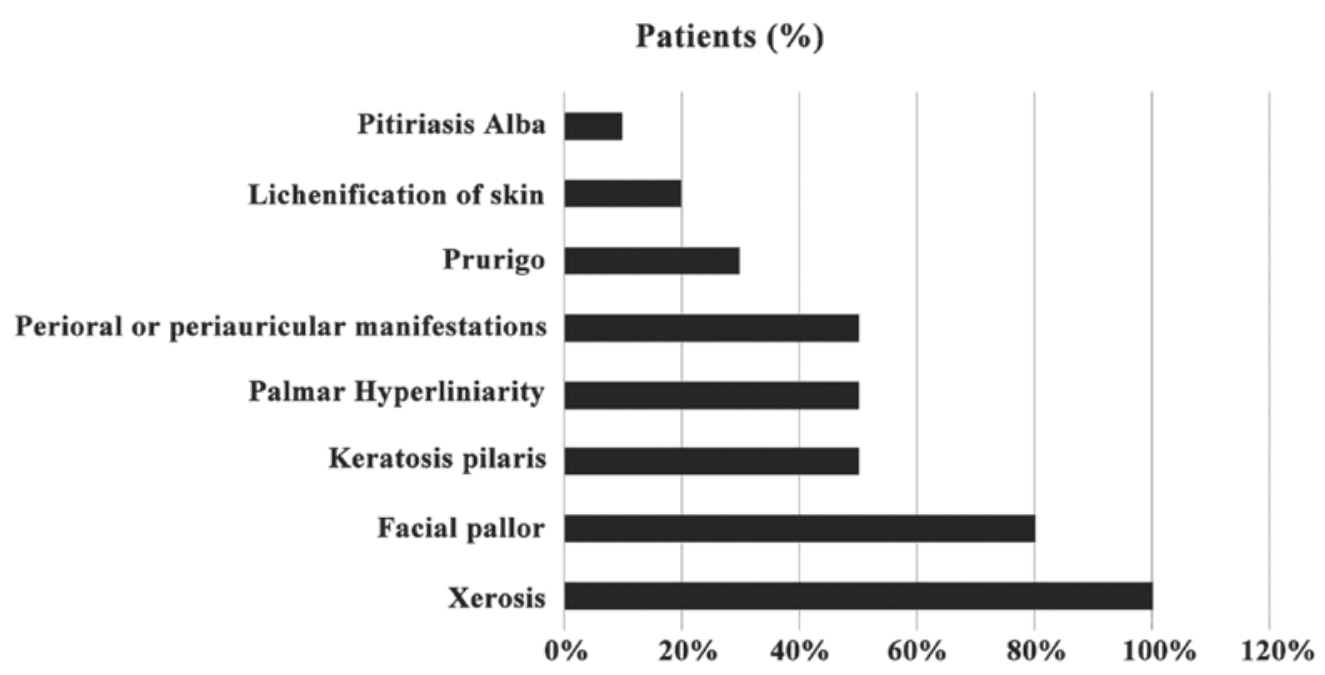

Figure 1. Clinical manifestations exhibited by the patients included in the study.

Questionnaire results. The median value for DLQI was 8 , with the 25 th percentile at 4.75 and the 75 th percentile at 15.75. Three of them were experiencing only minimal impact of $\mathrm{AD}$ on their lives (DLQI $<5$ ), four of them were moderately affected by the AD symptoms (DLQI 6-10) and the other three patients had their QoL seriously affected by AD (DLQI 11-20).

For the SCORAD questionnaire, the median value obtained was 35.05 , with the 25 th percentile at 24.03 and the 75 th percentile at 54.23 . Of the patients $30 \%$ presented mild AD (SCORAD, 25); other $40 \%$ registered SCORAD values between 25 and 50, being diagnosed with moderate AD. Of the patients $30 \%$ had a severe form of AD (SCORAD >50).

Ultrasound results. The non-lesional SLEB median was 13 , with the 25 th percentile at 0 and the 75 th percentile at 181 . The lesional SLEB median was 164 , with the 25 th percentile at 49.50 and 75 th percentile at 279.50 . The non-lesional skin thickness median was $875.50 \mu \mathrm{m}$, with the 25 th percentile at $622 \mu \mathrm{m}$ and the $75 \mathrm{th}$ percentile at $1409 \mu \mathrm{m}$. The lesional skin thickness had a median of $1099.50 \mu \mathrm{m}$, with the $25 \mathrm{th}$ percentile at $875.50 \mu \mathrm{m}$ and the $75 \mathrm{th}$ percentile at $1362 \mu \mathrm{m}$. The non-lesional intensity median was 60.4 , with 25 th and 75 th percentiles at 39.15 and 68.475 respectively. The lesional intensity score median was at 37.95 , with the 25 th and 75 th percentiles at 34.675 and 52.5, respectively.

Correlations. In non-lesional skin measurements, DLQI was correlated with skin thickness (Spearman's rho correlation coefficient $=0.657 ; \mathrm{P}=0.039$ ) and intensity (Spearman's rho $\mathrm{CC}=0.675, \mathrm{P}=0.032)$. The correlation of DLQI and SLEB was not statistically significant $(\mathrm{P}=0.215)$. The SCORAD score was not significantly for any of the investigated ultrasonographical markers: for SLEB, $\mathrm{P}=0.590$, for thickness $\mathrm{P}=0.511$, and for intensity, $\mathrm{P}=0.556$. The correlations of lesional skin can be found in Table I, but they did not reach statistical significance.

\section{Discussion}

The most valuable finding of our study is that DLQI is associated with the intensity and thickness of non-lesional skin. This confirms that indeed, AD affects the overall skin barrier, and this in itself causes an impact on the patients' QoL. This finding is sustained by the current literature: it has been established that apparently healthy skin presents subclinical disturbances and changes in patients suffering from AD (4). Furthermore, the images obtained through 
HF-USG correlate with histology results in the case of seemingly healthy skin, supporting the same conclusion: that there is subclinical change in the apparently healthy skin of patients with AD (18). We follow with the notion that these changes are meaningful enough to impact our patients' QoL. The SCORAD results did not significantly correlate with our ultrasonographic parameters, nor with the DLQI scores. This suggests that the perception of the condition by the patient might influence their QoL to a higher extent than the objective reality of their condition.

The strength of our study is that, to our knowledge, it is the first study in Romania that used HF-USG to assess changes in $\mathrm{AD}$ and correlated it with clinical and subjective parameters.

The major limitation to our study is the sample size. We expect that the enlargement of this initial study would attain a higher level of statistical significance.

With regards to our study group, it is skewed towards young female adults. Both male participants included in the study had BMIs outside of the reference range (one had a BMI of 17, classified as underweight and the other had a BMI of 33 classified as first degree obesity). A larger sample size is needed to determine whether AD is correlated with abnormal weight in men.

Most of our patients are a Fitzpatrick phototype II, with 1 patient being a I and 2 being a III. This limits the conclusions of our study to populations in Central-Northern Europe.

The medians of both DLQI and SCORAD classify as moderate in terms of impact on the QoL and severity, thus pointing out that our study results might not apply in patients with severe or mild forms of AD.

Our aim is to continue this project and extend our ultrasonographic evaluation (HF-USG) of AD patients, including patients with mild or severe forms of the condition, and crystallize the clinical and subjective image of the patient suffering from AD.

Our results offer a basis to reassess the current therapeutic approach, which for the moment focuses only on the remission of active lesions. It might be reasonable to consider targeting the overall state of the skin barrier, as well as its impact on the patients' QoL.

In conclusion, our study revealed that HF-USG of the skin is able to assess specific modifications of the AD, both in lesional and non-lesional areas. The hypoechoic band (SLEB) can be present even in the normal-looking, non-lesional skin of some AD, presenting barrier function defects and may indicate subclinical eczematous skin reactions, in early stages AD. In non-lesional skin, the measured thickness corelates with patients QoL.

As a noninvasive and objective evaluation, HF-USG could be included in the current management of $\mathrm{AD}$, helping assess disease severity and therapeutic outcome, in correlation with common scales and scores.

\section{Acknowledgements}

Not applicable.

\section{Funding}

No funding was received.

\section{Availability of data and materials}

The datasets used and/or analyzed during the current study are available from the corresponding author on reasonable request.

\section{Authors' contribution}

MS and RFI were responsible for the aquisition and analysis of the data regarding the disease and the HF-USG parameters. AT and ANB contributed to the conception and design of the study, and revising it critically for important intellectual content. All authors read and approved the final version of the manuscript.

\section{Ethics approval and consent to participate}

The study was approved by the Ethics Committee of 'Iuliu Hatieganu' University of Medicine and Pharmacy (Cluj-Napoca, Romania), and written informed consent was obtained from all patients.

\section{Patient consent for publication}

Not applicable.

\section{Competing interests}

The authors declare that they have no competing interests.

\section{References}

1. Lawrance FE: Guidelines of care for the management of atopic dermatitis-Part 1: Diagnosis and assessment of atopic dermatitis. J Am Acad Dermatol 70: 338-351, 2015.

2. Wollenberg A and Bieber T: Proactive therapy of atopic dermatitis - an emerging concept. Allergy 64: 276-278, 2009.

3. Eichenfield LF, Tom WL, Berger TG, Krol A, Paller AS, Schwarzenberger K, Bergman JN, Chamlin SL, Cohen DE, Cooper KD, et al: Guidelines of care for the management of atopic dermatitis: Section 2. Management and treatment of atopic dermatitis with topical therapies. J Am Acad Dermatol 71: 116-132, 2014.

4. Polańska A, Dańczak-Pazdrowska A, Silny W, Jenerowicz D, Olek-Hrab K and Osmola-Mańkowska A: Nonlesional skin in atopic dermatitis is seemingly healthy skin - observations using noninvasive methods. Wideochir Inne Tech Malo Inwazyjne 8: 192-199, 2013.

5. Polańska A, Dańczak-Pazdrowska A, Silny W, Jenerowicz D, Osmola-Mańkowska A and Olek-Hrab K: Evaluation of selected skin barrier functions in atopic dermatitis in relation to the disease severity and pruritus. Postepy Dermatol Alergol 29: 373-377, 2012.

6. Basra MK, Salek MS, Camilleri L, Sturkey R and Finlay AY: Determining the minimal clinically important difference and responsiveness of the Dermatology Life Quality Index (DLQI): Further data. Dermatology 230: 27-33, 2015.

7. Heinl D, Chalmers J, Nankervis H and Apfelbacher CJ: Eczema trials: Quality of life instruments used and their relation to patient-reported outcomes. A systematic review. Acta Derm Venereol 96: 596-601, 2016.

8. Rehal B and Armstrong AW: Health outcome measures in atopic dermatitis: A systematic review of trends in disease severity and quality-of-life instruments 1985-2010. PLoS One 6: e17520, 2011.

9. Holm JG, Agner T, Clausen ML and Thomsen SF: Quality of life and disease severity in patients with atopic dermatitis. J Eur Acad Dermatol Venereol 30: 1760-1767, 2016. 
10. Fornage BD: High-frequency sonography of the skin. Eur J Ultrasound 2: 173-182, 1995.

11. Gutierrez M, Wortsman X, Filippucci E, De Angelis R, Filosa G and Grassi W: High-frequency sonography in the evaluation of psoriasis: Nail and skin involvement. J Ultrasound Med 28: 1569-1574, 2009.

12. Marina ME, Botar Jid C, Roman II, Mihu CM and Tătaru AD: Ultrasonography in psoriatic disease. Med Ultrason 17: 377-382, 2015.

13. Polańska A, Dańczak-Pazdrowska A, Jałowska M, Żaba R and Adamski Z: Current applications of high-frequency ultrasonography in dermatology. Postepy Dermatol Alergol 34: 535-542, 2017.

14. Filipowska-Grońska A, Weryńska-Kalemba M, Bożek A, Filipowska B, Żebracka-Gala J, Rusinek D, Kula D and Jarząb J: The frequency of polymorphic variants of filaggrin gene and clinical atopic dermatitis. Postepy Dermatol Alergol 33: 37-41, 2016
15. Lifschitz C: The impact of atopic dermatitis on quality of life. Ann Nutr Metab 66: 34-40, 2015.

16. Rzany B: Too many instruments for measuring Quality of Life in Atopic Dermatitis. J Eur Acad Dermatol Venereol 31: 574-574, 2017.

17. Kong TS, Han TY, Lee JH and Son SJ: Correlation between severity of atopic dermatitis and sleep quality in children and adults. Ann Dermatol 28: 321-326, 2016.

18. Polańska A, Dańczak-Pazdrowska A, Silny W, Woźniak A, Maksin K, Jenerowicz D and Janicka-Jedyńska M: Comparison between high-frequency ultrasonography and histopathology in atopic dermatitis. Ski Res Technol 19: 432-437, 2013. 